protein BUBR1 (ref. 11). As the motor activity of CENP-E is dedicated to aligning mono-oriented chromosomes, its engagement acts as an indicator of unattached kinetochores. Thus, it is clear why CENP-E activity is directly linked to the checkpoint signalling pathway.

In summary, these data completely overturn previous models of chromosomal alignment during mitosis by showing that mono-oriented chromosomes move to the spindle equator before bi-orientation. Although this study shows how chromosomes reach the equator and achieve bi-orientation, the question of how chromosomes maintain alignment after bi-orientation remains open. Also, unattached kinetochores are laden with the microtubule motor dynein $^{12}$, which moves on microtubules in the opposite direction relative to CENP-E. In the face of apparent competition between these two motors, how does CENP-E dominate to move chromosomes toward the spindle equator? Finally, movement of a mono-oriented chromosome toward the spindle equator would tug on the kinetochore fibre that is guiding its movement. Does this tug affect the behaviour of the kinetochore associated with that kinetochore fibre? Errors in chromosome segregation are irreversible, so these data represent a large step toward understanding how chromosomes align and why chromosome alignment might fail. Given the high price that is paid for chromosome mis-segregation, it now seems reasonable that chromosomes should cooperate to ensure that they are all aligned before segregation.
1. Wittman, T., Hyman, A. \& Desai, A. Nature Cell Biol. 3, E28-E34 (2001)

2. Rieder, C. L. \& Salmon, E. D. J. Cell Biol. 124, 223-233 (1994).

3. Kapoor, T. M. et al. Science 311, 388-391 (2006).

4. Yen, T.J. et al. EMBO J. 10, 1245-1254 (1991).

5. Wood, K.W., Sakowicz, R., Goldstein, L.S.B. \& Cleveland, D. W. Cell 91, 357-366 (1997)

6. Khodjakov, A., Copenagle, L., Gordon, M. B., Compton, D.A. \& Kapoor, T.M.J. Cell Biol. 160, 671-683 (2003).

7. Lampson, M.A., Renduchitala, K., Khodjakov, A. \& Kapoor, T. Nature Cell Biol. 6, 232-237 (2004).

8. McEwen, B. F. et al. Mol. Biol. Cell 12, 2776-2789 (2001).

9. Pinsky, B.A. \& Biggins, S. Trends Cell Biol. 15, 486493 (2005).

10. Ahonen, L.J. et al. Curr. Biol. 15, 1078-1089 (2005).

11. Chan, G. K., Jablonskin, S. A., Sudakin, V., Hittle, J.C. \& Yen, T.J.J. Cell Biol. 146, 941-954 (1999).

12. Hoffman, D.B, Pearson, C. G., Yen, T. J., Howell, B. J. \& Salmon, E. D. Mol. Biol. Cell 12, 1995-2009 (2001).

\section{Eisosomes: endocytic portals}

Cells internalize plasma membrane receptors, soluble nutrients, lipids and other cargo by endocytosis. Reporting in the February 23 issue of Nature (Nature 439, 998; 2006), Peter Walter and colleagues describe structures called 'eisosomes', which mark hotspots of endocytosis at the yeast plasma membrane.

Although many components of the endocytic apparatus have been identified and studied in detail in yeast and other eukaryotes, the spatial regulation of endocytosis, that is, whether it occurs at specific predetermined locations or at random sites on the plasma membrane, remains poorly understood. The authors' began by showing that two proteins of unknown function, Pill and Lsp1, colocalize to several discrete spots at the cell cortex that they termed 'eisosomes'. They characterized these structures further and showed that they are largely immobile and were composed primarily of Pil1 and Lsp1.

As the authors found that Sur7 - a protein previously shown to interact genetically with the endocytic protein endophilin - localizes to eisosomes, they decided to examine whether eisosomes act in endocytosis. They found that both lipid and protein cargo localized to eisosomes when endocytosis was inhibited and that deleting the eisosome components Pill and Lsp1 reduced receptor endocytosis and resulted in an accumulation of aberrant plasma membrane invaginations in the Pill mutant. Moreover, perturbing eisosome distribution so that they formed large clusters resulted in concomitant redirection of endocytic cargo to these clusters, providing strong evidence that eisosomes mark sites of entry into the cell.

To understand whether, and how, eisosomes intersect with other known endocytic regulators, the authors focused on cortical actin

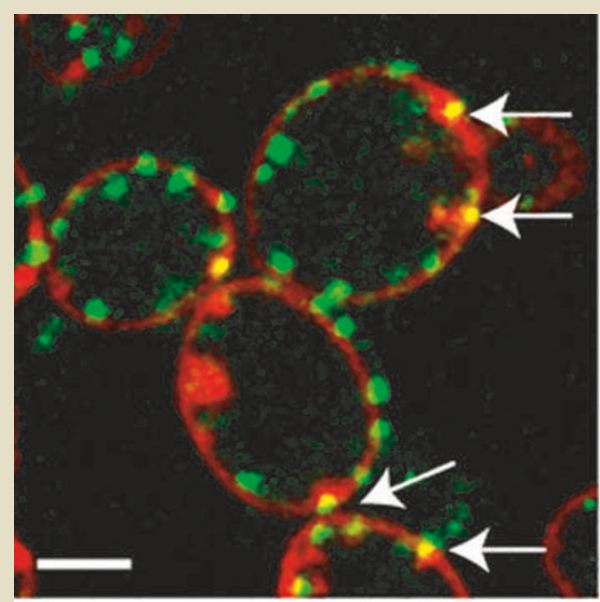

The lipid marker, FM4-64 (red) colocalizes with the eisosome component Pil1 (green). Foci representing endocytic intermediates containing Pil1 and FM4-64 are shown in yellow

patches, which have been shown to have an important function in endocytosis by facilitating endocytic vesicle formation. At least a subset of actin patches formed at eisosomes and although actin did not appear to be important for recruiting endocytic cargo to eisosomes, it was required for uptake of cargo from these structures. Finally, the authors described a set of genetic interactions between components of eisosomes and the endocytic apparatus providing additional evidence that eisosomes direct endocytosis.

Although this study places eisosomes in the yeast endocytic pathway, whether eisosome-like structures also direct endocytosis at specific sites in higher eukaryotes and how eisosome assembly is restricted to discrete locations has yet to be explained.

SOWMYA SWAMINATHAN 